\title{
The Effects of Topical Sesame (Sesamum indicum) Oil on Pain Severity and Amount of Received Non-Steroid Anti-Inflammatory Drugs in Patients With Upper or Lower Extremities Trauma
}

\author{
Marzieh Beigom Bigdeli Shamloo ${ }^{1}$; Morteza Nasiri ${ }^{2,}$; Aazam Dabirian ${ }^{3}$; Ali Bakhtiyari ${ }^{4}$; \\ Faraz Mojab ${ }^{5}$; Hamid Alavi Majd ${ }^{6}$ \\ ${ }_{2}^{1}$ Department of Nursing, Nursing and Midwifery School, Dezful University of Medical Sciences, Dezful, Iran \\ ${ }_{3}^{2}$ Department of Nursing, Nursing and Midwifery School, Ahvaz Jundishapur University of Medical Sciences, Ahvaz, Iran \\ ${ }^{3}$ Department of Pharmacognosy, Nursing and Midwifery School, Shahid Beheshti University of Medical Sciences, Tehran, Iran \\ ${ }_{5}^{4}$ Department of Emergency Medicine, Medicine School, Dezful University of Medical Sciences, Dezful, Iran \\ ${ }^{5}$ Pharmacy School, Shahid Beheshti University of Medical Sciences, Tehran, Iran \\ 6 Department of Epidemiology and Statistic, Health School, Shahid Beheshti University of Medical Sciences, Tehran, Iran \\ ${ }^{*}$ Corresponding author: Morteza Nasiri, Department of Nursing, Nursing and Midwifery School, Ahvaz Jundishapur University of Medical Sciences, Ahvaz, Iran. Tel: + 98-9171745485, \\ Fax:+98-7726223012, E-mail: mortezanasiri.or87@yahoo.com
}

Received: November 2, 2014; Revised: November 27, 2014; Accepted: January 7, 2015

\begin{abstract}
Background: Most patients with trauma experience different levels of pain. Due to side effects as well as economic burden of drugs used for pain relief after trauma commonly, it is important to use low-cost methods independently or combined with drugs to alleviate pain. Objectives: Therefore, this study aimed to investigate the effects of topical sesame oil on pain severity and frequency of received NSAIDs of patients with trauma.

Patients and Methods: This randomized clinical trial study was conducted on 150 patients with upper or lower extremities trauma in Dezful Ganjavian Hospital, Ahvaz, Iran, in 2014. Data was collected by a researcher-made questionnaire and Visual Analogue Scale (VAS). Patients were divided into two groups of control $(n=75)$ and intervention $(n=75)$ randomly. In the intervention group, patients applied topical sesame oil beside the routine cares, while in the control group patients just received routine cares. Severity of pain and frequency of received NSAIDs was assessed in the first, third, seventh and tenth days after the intervention in the both groups. Data was analyzed by SPSS19 software using descriptive and analytic (Chi-square and independent sample t-test) statistical methods.

Results: Based on student sample t-test, there was a significant difference between intervention and control groups regarding the pain severity in the first $(\mathrm{P}=0.06)$, third $(\mathrm{P}=0.001)$, seventh $(\mathrm{P}=0.001)$ and tenth $(\mathrm{P}=0.001)$ days after the intervention. Besides, the frequency of received NSAIDs in the intervention group and the control group showed significant difference in four days after the intervention (for four days $\mathrm{P}=0.001$ ).

Conclusions: Topical application of sesame oil could reduce pain severity and frequency of received NSAIDs in patients with upper or lower extremities trauma. Therefore, it is recommended to use this oil in complementary medicine for pain relief due to low cost, easy usage and lack of adverse effects.
\end{abstract}

Keywords: Sesame Oil; Pain Severity; Non-Steroid; Anti-Inflammatory Drugs; Trauma

\section{Background}

Trauma is a main cause of long-lasting morbidity and disability in individuals younger than 45 years and considered as the single greatest cause of years of life lost in the world $(1,2)$. Trauma, in addition to socioeconomic costs on the society and long-term medical needs, is the fourth leading cause of death in the world (3). Today, studies have shown increasing rate of trauma, especially in the U.S $(4,5)$. In developing countries, most deaths are caused by trauma as well $(2,6-8)$. Iran is a country with a high rate of road traffic crash fatality and injury. According to one epidemiological study conducted on Shahid Beheshti Medical Center, Kashan, Iran, 71.7\% of patients had multiple traumas (6).
Although today trauma associated mortality has decreased over time due to improved medical care and technical development, a proportion of patients may experience different levels of pain after trauma $(9,10)$. Investigations have shown that at one year after trauma, $62.7 \%$ of patients report trauma-related pain in at least one body region (11). Pain after trauma often exacerbates psychological distress (9) and diminishes quality of life (12). Therefore, control of pain is one of the necessary and essential nursing cares in patients with trauma.

Currently, Non-Steroid Anti-Inflammatory Drugs (NSAIDs) and opioids are the preferred drugs of choice for mild to moderate and severe pains respectively after

Copyright (C) 2015, Iranian Society of Regional Anesthesia and Pain Medicine(ISRAPM). This is an open-access article distributed under the terms of the Creative Commons Attribution-NonCommercial 4.0 International License (http://creativecommons.org/licenses/by-nc/4.0/) which permits copy and redistribute the material just in noncommercial usages, provided the original work is properly cited. 
trauma (13). However, these drugs improve patients' outcomes and reduce the incidence of post-traumatic pain, these can impose a lot of cost to patients and health care system and cause several undesirable adverse effects $(14,15)$. There is an increased risk in gastrointestinal bleeding, acute liver failure, renal toxicity, cardiovascular events, dizziness, nausea and vomiting when using NSAIDs (16). In addition, treatment with opioids is associated with a number of common adverse effects, including constipation, nausea or vomiting, pruritus, cognitive impairment, dry mouth, tolerance or dependence and urinary retention (17). Therefore, it is important to design a new low-cost appropriate method independently or combined with palliatives for the management of posttraumatic pain and consequently preventing undesirable drugs adverse effects.

To date, studies have shown a need for alternative and complementary therapy for pain management after trauma $(18,19)$. Currently, therapeutic effects of some herbal products such as comfrey root (Symphytum officinale) extract are discussed in researches for short-term management of pain after ankle trauma (20-22). Sesame oil has been another herbal extract useful as external administration in management of pain in previous studies (23-25).

Sesame oil is derived from the plant species Sesamum indicum L., an herbaceous annual belonging to the Pedaliaceae family (26). Sesame oil has been long used in traditional medicine of Iran and many other countries due to its antioxidant, anti-inflammatory and anti-bacterial significant effects (27). In traditional Taiwanese medicine, sesame oil was used to relief pain in people with joint pain, toothache, premenstrual syndrome, scrapes and cuts (23). Recently, sesame oil has been proved to possess a broad spectrum pharmacological effects including anti-mutagenic, anti-oxidant, anti-cardio, anti-inflammatory and anti-pyretic protective effects as well as anti-nociceptive property (23-25). In experimental animal models, this kind of oil exerted analgesic activity (23-25), but until now there have been no human studies to evaluate the effect of topical sesame oil on pain management in patients.

\section{Objectives}

Considering the importance of pain management after trauma and regarding the adverse effects of opioids and NSAIDs used for pain management after trauma, we aimed to investigate the effects of topical sesame oil on pain severity and frequency of received NSAIDs of patients with upper or lower extremities trauma admitted to Dezful Ganjavian hospital, Ahvaz, Iran, to find a novel approach in management of pain after trauma.

\section{Patients and Methods}

\subsection{Study Design}

This randomized clinical trial study was conducted on 150 patients with upper or lower limbs trauma referred to emergency department of Dezful Ganjavian Hospital, Ahvaz, Iran, from March first through September 30th, 2014. All patients with one or multiple blunt trauma in upper extremity (finger, wrist, lower arm, elbow, upper arm) or lower extremity (toe, foot, ankle, lower leg, knee, upper leg and lower trunk) occurred at least 1 hour and maximally 6 hours after admission, were entered the study. Inclusion criteria were age range of 15 - 45 years, Body Mass Index (BMI) of $18-30 \mathrm{~kg} / \mathrm{m}^{2}$, full consciousness and being able to understand study details, lack of any sign of bone fractures, internal or external bleeding, dislocation, amputation, presence of a foreign body, nerve damage, fever as well as infection, not using cast or splint at trauma site, having regional and moderate pain based on Visual Analog Scale (VAS) (obtaining score between 3 - 6), lack of any history of addiction, cigarette and alcohol abuse, lack of any sensitivity or allergy to the sesame plant group, not receiving drugs or herbal extracts which may interact with the study therapeutic protocol such as anti-coagulant and analgesics and free from diseases that may affect the pain severity such as diabetes, cardiovascular, liver, kidney and musculoskeletal diseases. Patients with any sing of allergy to the sesame oil or peptic or duodenal ulcers, and those who preferred to receive the treatment out of the study were excluded. Besides, inappropriate followup by patients (missing follow-up more than two times) and patient's desire to withdraw in any phase of the study were considered as other exclusion criteria.

\subsection{Sample Size}

To estimate the sample size, a pilot study was conducted on 10 patients (not included in the main sample). Based on the results and using the sample size formula of RCT study with confidence level of $95 \%$, the number of needed samples was calculated as 63 patients; we considered 75 subjects in each group for getting more confident results.

\subsection{Data collection}

Data was collected based on interview and patients' records using a researcher-made questionnaire and Visual Analogue Scale(VAS). The researcher-made questionnaire assessed demographic and clinical characteristics of patients including age, gender, marital status, education level, occupation, BMI, site of trauma, size of trauma, complications of trauma and received NSAIDs. In this study, BMI was calculated by measuring patients' height and body weight and using the following equation;

$$
\mathrm{BMI}=\frac{\text { weight }}{(\text { height })^{2}}
$$

as $\mathrm{Kg} / \mathrm{m}^{2}$. To determine the scientific validity of this questionnaire, content validity was performed. For this purpose, after studying books and other resources re- 
lated to this subject, a checklist was prepared and then presented to 10 faculty members of nursing school of International Branch of Shahid Beheshti University of Medical Sciences, Iran, and after collecting their suggestions, the final checklist was prepared. For measuring the pain severity, we used VAS. This instrument is for subjective characteristics or attitudes cannot be directly measured. When responding a VAS item, respondents specified their level of agreement to a statement by indicating a position along a continuous line between two end-points ( 0 and 10). Zero and 10 are considered as lack of pain and the maximum severity of pain, respectively. Based on this scale, 0 - 3 scores are considered as mild pain, 3 - 6 scores as moderate pain and 6 - 10 scores as severe pain. This scale was used for measuring pain of patients with musculoskeletal trauma in different studies and its reliability and validity were proven in previous studies $(28,29)$. Size of trauma was measured based on area sensitive to pain using a scaled ruler designed according to square $\mathrm{cm}$. The incidence of adverse events was evaluated by recording all observed or volunteered adverse events. For this purpose, study related adverse events such as any sign of allergy or sensitivity, bleeding and infection were monitored by daily evaluation. For patients who withdrew or lost to follow-up, adverse events were acquired by telephone.

\subsection{Interventions}

This study was approved by the ethics committee of International Branch of Shahid Beheshti University of Medical Sciences, Iran, with code No. 116-397 and was registered in the Iranian Registry of Clinical Trials (IRCT) with code No. IRCT2013100714930N1. After obtaining an introduction letter from this committee and representing it to Dezful University of Medical Sciences, Iran and obtaining an informed consent from all patients and providing verbal explanation about the research and assurance of confidentiality and anonymity, patients were randomly allocated to intervention $(\mathrm{n}=75)$ and control $(\mathrm{n}=$ 75) groups using envelopes containing numbers from a table of random numbers.

At initial meeting considered as baseline (at the 1st day), assessment of patients' trauma, pain severity and frequency of received NSAIDs was performed for the both groups by the same trained specialist. Then, all patients were educated how to take care of their trauma in one section during two hours. For this purpose, both the studied groups were educated to put cold compress on the trauma site at the first day and put warm compress on the following days and taking just prescribed drugs (NSAIDs) and not to use any other medicine or herbal extracts for pain management. After that, patients in the intervention group received topical sesame oil in addition to routine cares; whereas, patients in the control group just received routine cares.

In the present study, routine cares were implemented based on cares protocol for patients with trauma devel- oped by physicians and nurses of the recruitment center. According to this protocol, the periphery and the center of the trauma site were irrigated and cleaned with $1000 \mathrm{~mL}$ sterile normal saline solution (0.9\%), and then dried with a sterile gaze and after that massaged in a circle way about 5 - 7 minutes based on the trauma size if needed. Furthermore, patients received NSAIDs including Diclofenac (Tab $50 \mathrm{mg}$ ), Ibuprofen (Tab $400 \mathrm{mg}$ ) and Indomethacin (Cap $25 \mathrm{mg}$ ) at certain intervals according to the medication dosage instruction and when needed. NSAIDs were administered via oral by a nurse of recruitment center, who was not aware of the groups assignment. Furthermore, it was instructed to patients in the both groups to put cold and warm compress on the trauma site when needed.

In this study, sesame oil (with $100 \%$ purity) manufactured by the Saman Sesame Oil Ltd. (Saman, Iran) was applied to trauma site in the intervention group. The external administration of this kind of sesame oil had not shown any adverse effects in previous study (30). Furthermore, based on our pilot study conducted on 10 patients (not included in main sample), the topical usage of this oil showed no adverse effects. For topical usage of oil, at first periphery and the center of the trauma site were irrigated and cleaned with $1000 \mathrm{~mL}$ sterile normal saline solution $(0.9 \%)$ and dried with sterile gauze, then 10 drops (about $1 \mathrm{~mm}$ thick and $3.8 \mathrm{~mL}$ ) of sesames oil was applied for each 50 square $\mathrm{cm}$ on the site of trauma, using a dropper. After that, site of trauma was massaged.

The intervention was performed once a day until 10 days in the both groups by the same trained and experienced researcher in the recruitment center or patients' home. At the end of the third, seventh and tenth days of followup, the pain severity and frequency of received NSAIDs were assessed and scored again with previous questionnaire in the both groups by the same trained specialist. Both pre- and post-assessment and scoring were performed by the same trained specialist not aware of the groups assignment. Besides, the statistical analyzer was unaware of groups assignment.

\subsection{Statistical Analysis}

Statistical analysis was performed using the Statistical Package for the Social Sciences (SPSS Inc., Chicago, IL, USA) version 19). Descriptive statistical tests, mean and standard deviation frequency and percentage) were used for demographic and clinical characteristics. Independent sample t-test was used to compare quantitative variables between the both groups. Furthermore, Chi-square and Mann-Whitney tests were performed for qualitative variables. $\mathrm{P}<0.05$ was considered as statistical significance.

\section{Results}

\subsection{Demographic and Baseline Characteristics}

of 150 patients enrolled in this study, 126 adhered to the 
protocol. Nine patients in the control group and fifteen patients in the intervention group were excluded from the study due to loss of follow-up. Therefore, 126 patients (63 patients in the intervention group and 63 patients in control group) were included in the final analysis. The flow chart of patients' recruitment is shown in Figure 1.

The patients' age in the both studied groups was ranged from 25 - 35 years with a means age of $33.8 \pm 1.3$ and $32.6 \pm$ 9.13 years for the intervention and control groups, respectively. Based on student's sample t-test, there was no significant difference regarding the patients' age between the groups $(\mathrm{P}=0.877)$. Other demographical and clinical characteristics of patients are shown in Table 1, indicating no statistically significant differences between the two groups $(\mathrm{P}>0.5)$.

\subsection{Pain Severity}

The data in Table 2 shows pain severity between the intervention and control groups during the 1st, 3rd, 7th and 10th days of follow-up. Based on this table, independent t-test indicated no significant difference between the two groups regarding the pain severity at first day $(\mathrm{P}=0.06)$, while at 3rd, 7th and 10th days of follow-up, a significant difference was found (for three days $\mathrm{P}=0.001$ ).

\subsection{NSAIDs Use}

The data in Table 3 shows frequency of received NSAIDs between the intervention and control groups during the 1st, 3rd, 7th and 10th days of follow-up. Based on this table, results indicated no significant difference between the two groups regarding NSAIDs use at first day $(P=0.66)$, while at 3rd, 7th and 10th days of follow-up, a significant difference was found (for three days $\mathrm{P}=0.001$ ).

\subsection{Adverse Effects}

All patients tolerated treatment by topical sesame oil very well and there were no adverse effects to report during the study.

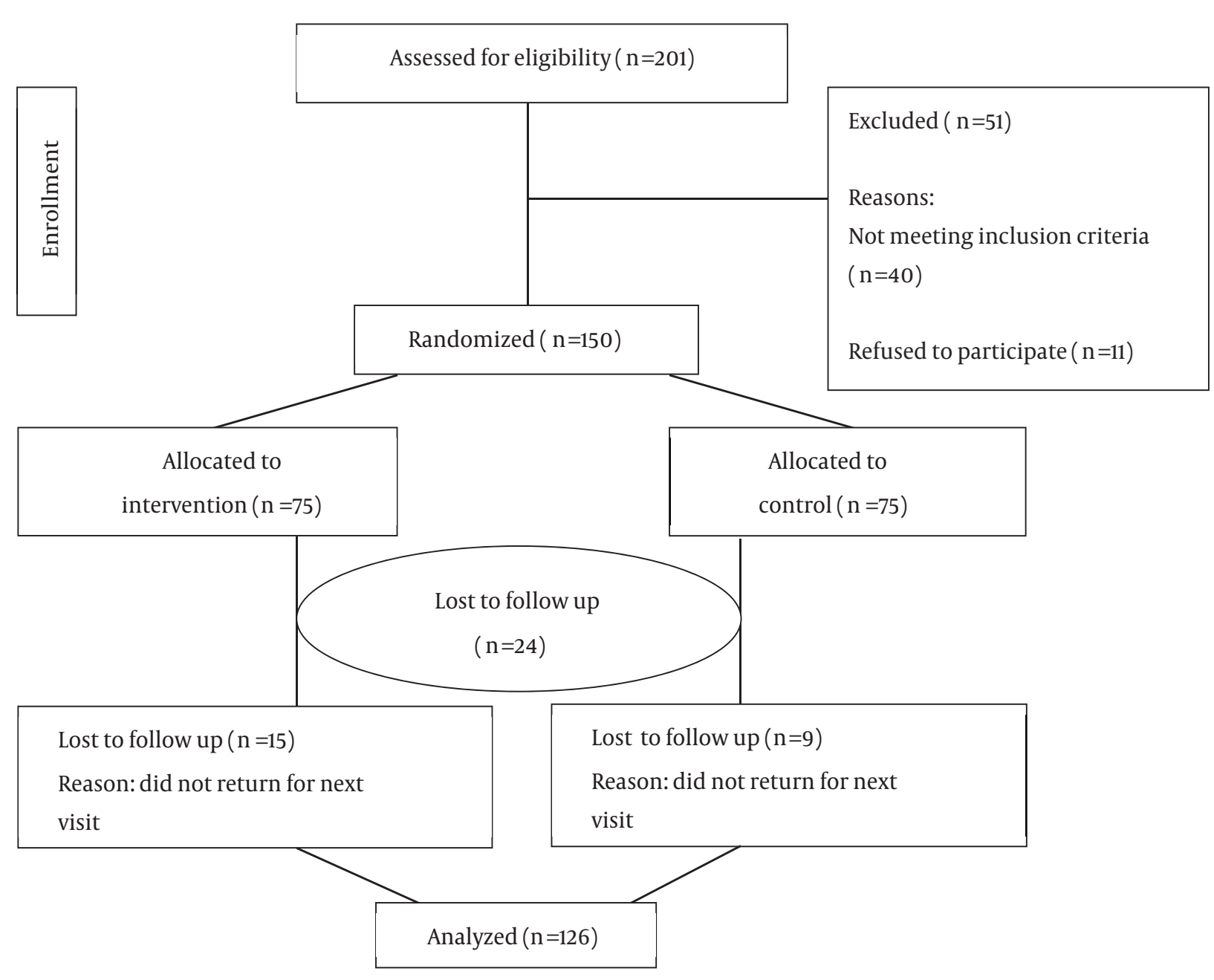

Figure 1. Flow of Participants in the Trial 
Bigdeli Shamloo MB et al.

Table 1. Demographic and Clinical Characteristic of Patients in the Intervention and Control Groups a

\begin{tabular}{|c|c|c|c|}
\hline Demographic Characteristic/Group & Intervention $(n=63)$ & $\operatorname{Control}(n=63)$ & PValue \\
\hline Gender & & & 0.605 \\
\hline Female & $38(60.31)$ & $35(55.55)$ & \\
\hline Male & $25(39.69)$ & $28(44.45)$ & \\
\hline Educational level & & & 0.62 \\
\hline Illiterate & $9(14.28)$ & $8(12.70)$ & \\
\hline Less than diploma & $17(26.98)$ & $15(23.81)$ & \\
\hline Diploma & $10(15.88)$ & $16(25.39)$ & \\
\hline Collegiate & $27(42.86)$ & $24(38.10)$ & \\
\hline Marital status & & & 0.68 \\
\hline Married & $46(73.02)$ & $48(7619)$ & \\
\hline Divorced & $17(26.98)$ & $15(23.81)$ & \\
\hline Occupation & & & 0.69 \\
\hline Housewife & $17(26.98)$ & $17(26.98)$ & \\
\hline $\begin{array}{l}\text { Working (unskilled, skilled and clerical } \\
\text { work) }\end{array}$ & $29(46.04)$ & 25 (39.69) & \\
\hline Not working & $17(26.98)$ & $21(33.33)$ & \\
\hline BMI kg/m² & & & 0.89 \\
\hline$<18$ & $10(15.88)$ & $9(14.28)$ & \\
\hline $18.1-25$ & $33(52.38)$ & $37(58.73)$ & \\
\hline $25.1-30$ & $12(19.04)$ & $11(17.46)$ & \\
\hline$>30$ & $8(12.70)$ & $6(9.53)$ & \\
\hline Site of trauma & & & 00.08 \\
\hline Upper extremity Left region & $16(25.39)$ & $19(30.15)$ & \\
\hline Upper extremity Right & $19(30.15)$ & $10(15.88)$ & \\
\hline Lower extremity Left & $7(11.12)$ & $3(4.76)$ & \\
\hline Lower extremity Right & $21(33.34)$ & $31(49.21)$ & \\
\hline Size of trauma, $\mathrm{cm}^{2}$ square & & & 0.08 \\
\hline$<50$ & $27(42.86)$ & $14(22.22)$ & \\
\hline $51-100$ & $27(42.86)$ & $37(58.73)$ & \\
\hline $101-150$ & $8(12.70)$ & $9(14.28)$ & \\
\hline $151-200$ & $1(1.58)$ & $3(4.77)$ & \\
\hline Complications of trauma & & & 0.36 \\
\hline Yes Erosion & $8(12.70)$ & $7(11.12)$ & \\
\hline Yes Ecchymosis & $27(42.86)$ & $33(52.38)$ & \\
\hline No & $28(44.44)$ & $23(36.50)$ & \\
\hline Kind of NSAIDs use & & & 0.06 \\
\hline Diclofenac, Tab 50 mg & $11(17.46)$ & $4(6.35)$ & \\
\hline Ibuprofen, Tab $400 \mathrm{mg}$ & $51(80.96)$ & $58(92.07)$ & \\
\hline Indomethacin, Cap 25 mg & $1(1.58)$ & $1(1.58)$ & \\
\hline
\end{tabular}


Bigdeli Shamloo MB et al.

Table 2. Comparison of Pain Severity Between the Intervention and Control Groups During the 1st, 3rd, 7th and 10th Days of Followup

\begin{tabular}{lccc}
\hline Day & \multicolumn{2}{c}{ Mean/SD } & \multirow{2}{*}{ P Value } \\
\cline { 2 - 4 } & Intervention & Control & 0.06 \\
\hline 1st & $6.86 / 1.59$ & $6.44 / 1.63$ & 0.001 \\
3rd & $4.95 / 1.40$ & $5.83 / 1.58$ & 0.001 \\
7th & $2.78 / 1.32$ & $4.32 / 1.67$ & 0.001 \\
\hline 10th & $1.14 / 1.43$ & $2.83 / 1.53$ & \\
\hline
\end{tabular}

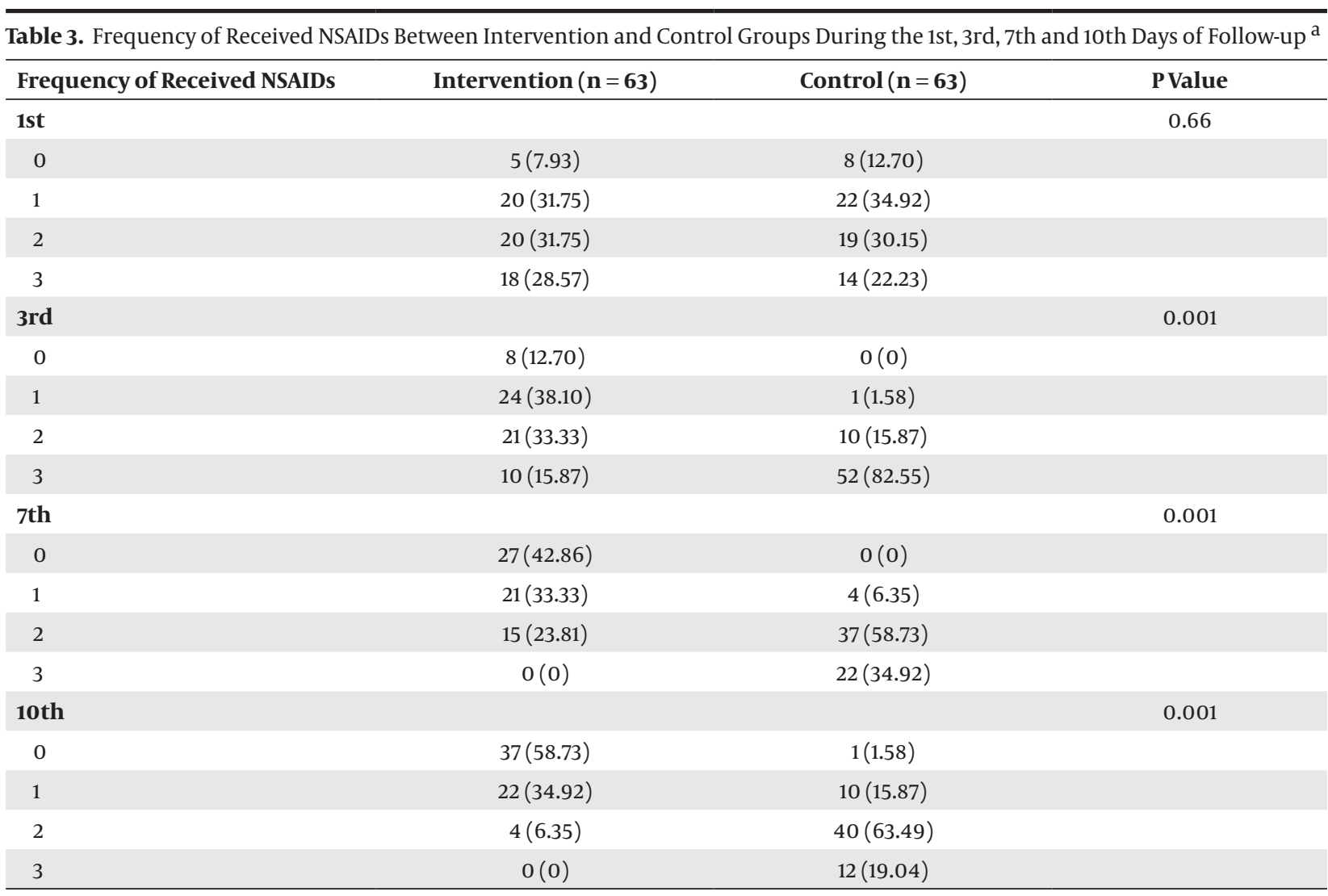

a Data are presented as No.(\%).

\section{Discussion}

Considering that using NSAIDs and opioids exerts a wide range of adverse effects $(14,15)$, there is currently a strong interest in developing new therapeutic agents from natural products to inhibit mediators involved in the evolution of pain (31). Although some herbal extracts have been reported to alleviate pain in musculoskeletal disorders $(18,19)$, to the best of our knowledge, this study is one of the first investigations conducted to investigate the effects of topical sesame oil on pain severity and frequency of received NSAIDs of patients with trauma.

Our results showed that application of topical sesame oil is effective to reduce pain severity and decrease the frequency of received NSAIDs. Unfortunately, most founded studies regarding our subject were performed in animal models and we could not find any similar study to compare our results. In one research conducted by Eftekhar Sadat et al. to assess the effect of administration of sesame seed supplementation on clinical signs and symptoms in patients with knee osteoarthritis (OA), a significant difference in pain intensity between the two groups after treatment was observed $(\mathrm{P}=0.004)(29)$, which is consistent with our results. Hsu et al. showed the effectiveness of sesame oil on relief of pain and inflammation in experimental gout model (23). In another study conducted by Hirsch et al. to compare the effect of Sesamum indicum herbal ointment and Flamazine for treating superficial burns, results showed pain relief after burns (32). Monteiro et al. revealed that sesame oil was able to 
decrease the time that the animal spent licking the injected paw on the first phase (due to a direct effect on nociceptors) and the second phase (due to an inflammatory response that involves different chemical mediators) of pain (25). Centrally acting drugs such as opioids inhibit both phases equally, but peripherally acting drugs, such as aspirin, indomethacin and dexamethasone, inhibit only the second phase (24). Therefore, sesame oil has probably similar action to the opioids and acts better than NSAIDs for pain relief. However, Monteiro et al. demonstrated that sesame oil exerted significant prolongation in the response latency time to the heat stimulus as a well-validated model for detection of opiate analgesics as well as several types of analgesic drugs in animal models and therefore, produced a similar effect to morphine that significantly increased the latency time to the nociceptive response compared with the control group (25).

Despite positive clinical evidence, the pathophysiological basis of sesame oil benefits is not yet clear. It is known that sesame oil has a high content of unsaturated fatty acids (palmitic, stearic, oleic and linoleic acids), lignans (sesamin, asarinin, sesamolin and sesamol) and gammatocopherol responsible for pain relief (33). Several studies indicated that fatty acids reduce the levels of prostaglandins and leukotrienes and thus decrease the pain (34, 35). Palmitic acid for example, showed to decrease the thermal nociception in mice (34). In addition, lignans such as sesamin have been described to relieve pain (36). In this study, we used Saman brand of sesame oil, which the previous study (27) indicated highest content of sesamol lignans in this brand. Based on recent investigation, sesamin is one of the active compounds in sesame oil and justify the antinociceptive and anti-inflammatory properties of this product (25). However, further studies are necessary to understand the mechanisms of action and correlate pharmacological activity with chemical composition of sesame oil.

A major concern during the treatment with herbal medications is unpredicted adverse effects such as allergic reactions. Fortunately similar to the Nekuzad et al. study, which showed that external use of sesame oil was safe and well-tolerated for prophylaxis from phlebitis (30), no significant adverse effects were observed in this study for patients treated with sesame oil indicating that this oil could well become a part of routine therapy for pain relief after trauma. However, further studies should be conducted to ensure the safety, feasibility and sustainability of usage.

Based on our results, it is recommended to use this oil in complementary medicine for pain relief, because it is found abundantly in Iran and has no adverse effects compared to chemical drugs, also is quite cheap. The finding of the present study can also be used as a step towards other researches in educational and research centers.

Some limitations in this study should be noted. Firstly, patient response to pain is affected by genetic differences that were out of researchers' control. Secondly, due to the waxy nature of sesame oil and the opinion of the supervisor pharmacologist, we could not use placebo in the control group. Finally, most founded studies regarding our subject were performed on animal models and we could not compare our results with other human researches. Therefore, it is suggested to pay more attention to these limitations and conduct investigations on human in future studies.

\section{Acknowledgements}

We appreciate the Research Deputy vice-chancellor for research affairs of International Branch of Shahid Beheshti University of Medical Sciences, Iran, for technical support as well as valuable assistance of all participants. We also sincerely thank the staff of Ganjavian hospital and department Nursing of Dezful University of Medical Sciences.

\section{Authors' Contributions}

Marzieh Beigom Bigdeli Shamloo and Morteza Nasiri devised the study concept, developed the study design, collected data, performed the study intervention, involved in the study conception and performed the analyzes and final preparation of the manuscript Aazam Dabirian and Faraz Mojab participated as advisor and supervised data collection and analysis. Ali Bakhtiyari assisted in data gathering and involved in study coordination and manuscript revision. Hamid Alavi Majd participated as supervisor and did statistical analysis. All authors read and approved the final manuscript.

\section{Funding/Support}

This study was adopted from MSc dissertation of Beigom Bigdeli Shamloo M, supported by International Branch of Shahid Beheshti University of Medical Sciences, Iran.

\section{References}

1. Probst C, Pape HC, Hildebrand F, Regel G, Mahlke L, Giannoudis P, et al. 30 years of polytrauma care: An analysis of the change in strategies and results of 4849 cases treated at a single institution. Injury. 2009;40(1):77-83.

2. Norouzi V, Feizi I, Vatankhah S, Pourshaikhian M. Calculation of the probability of survival for trauma patients based on trauma score and the injury severity score model in fatemi hospital in ardabil. Arch Trauma Res. 2013;2(1):30-5.

3. Mock C, Cherian MN. The global burden of musculoskeletal injuries: challenges and solutions. Clin Orthop Relat Res. 2008;466(10):2306-16.

4. Ootes D, Lambers KT, Ring DC. The epidemiology of upper extremity injuries presenting to the emergency department in the United States. Hand (NY). 2012;7(1):18-22.

5. Lambers K, Ootes D, Ring D. Incidence of patients with lower extremity injuries presenting to US emergency departments by anatomic region, disease category, and age. Clin Orthop Relat Res. 2012;470(1):284-90.

6. Adib-Hajbaghery M, Maghaminejad F. Epidemiology of patients with multiple trauma and the quality of their prehospital respiration management in kashan, iran: six months assessment. Arch Trauma Res. 2014;3(2):e17150. 
7. Hanche-Olsen TP, Alemu L, Viste A, Wisborg T, Hansen KS. Trauma care in Africa: a status report from Botswana, guided by the World Health Organization's "Guidelines for Essential Trauma Care". World J Surg. 2012;36(10):2371-83.

8. Fraga GP, Quintas ML, Abib Sde C. Trauma and emergency: is the unified health system (SUS) the solution in Brazil? Rev Col Bras Cir. 2014;41(4):232-3.

9. Rosenbloom BN, Khan S, McCartney C, Katz J. Systematic review of persistent pain and psychological outcomes following traumatic musculoskeletal injury. J Pain Res. 2013;6:39-51.

10. Castillo RC, MacKenzie EJ, Wegener ST, Bosse MJ, Leap Study Group . Prevalence of chronic pain seven years following limb threatening lower extremity trauma. Pain. 2006;124(3):321-9.

11. Rivara FP, Mackenzie EJ, Jurkovich GJ, Nathens AB, Wang J, Scharfstein DO. Prevalence of pain in patients 1 year after major trauma. Arch Surg. 2008;143(3):282-7.

12. Kaske S, Lefering R, Trentzsch H, Driessen A, Bouillon B, Maegele M, et al. Quality of life two years after severe trauma: A single centre evaluation. Injury. 2014;45 Suppl 3:S100-5.

13. Dijkstra BM, Berben SA, van Dongen RT, Schoonhoven L. Review on pharmacological pain management in trauma patients in (pre-hospital) emergency medicine in the Netherlands. Eur $J$ Pain. 2014;18(1):3-19.

14. Crofford LJ. Adverse effects of chronic opioid therapy for chronic musculoskeletal pain. Nat Rev Rheumatol. 2010;6(4):191-7.

15. Pountos I. Nonsteroidal anti-inflammatory drugs: prostaglandins, indications, and side effects. IJIM. 2011;3(1):19-27.

16. Teslim OA. Side Effects of Non-Steroidal Anti-Inflammatory Drugs: The Experience of Patients with Musculoskeletal Disorders. American J Health Res. 2014;2(4):106.

17. Labianca R, Sarzi-Puttini P, Zuccaro SM, Cherubino P, Vellucci $\mathrm{R}$, Fornasari D. Adverse effects associated with non-opioid and opioid treatment in patients with chronic pain. Clin Drug Invest. 2012;32(1):53-63.

18. Cantor JB, Gumber S. Use of Complementary and Alternative Medicine in Treating Individuals with Traumatic Brain Injury. Curr Phys Med Rehabil Rep. 2013;1(3):159-68.

19. Ernst E, Chrubasik S. Phyto-anti-inflammatories. A systematic review of randomized, placebo-controlled, double-blind trials. Rheum Dis Clin North Am. 2000;26(1):13-27.

20. Kucera M, Barna M, Horacek O, Kovarikova J, Kucera A. Efficacy and safety of topically applied Symphytum herb extract cream in the treatment of ankle distortion: results of a randomized controlled clinical double blind study. Wien Med Wochenschr. 2004;154(21-22):498-507.

21. Koll R, Buhr M, Dieter R, Pabst H, Predel HG, Petrowicz O, et al. Efficacy and tolerance of a comfrey root extract (Extr. Rad. Symphyti) in the treatment of ankle distorsions: results of a multicenter, randomized, placebo-controlled, double-blind study. Phytomedicine. 2004;11(6):470-7.
22. Predel HG, Giannetti B, Koll R, Bulitta M, Staiger C. Efficacy of a comfrey root extract ointment in comparison to a diclofenac gel in the treatment of ankle distortions: results of an observer-blind, randomized, multicenter study. Phytomedicine. 2005;12(10):707-14

23. Hsu DZ, Chen SJ, Chu PY, Liu MY. Therapeutic effects of sesame oil on monosodium urate crystal-induced acute inflammatory response in rats. Springerplus. 2013;2:659.

24. Saleem TSM, Basha SD, Mahesh G, Rani PVS, Kumar NS, Chetty CM. Analgesic, anti-pyretic and anti-inflammatory activity of dietary sesame oil in experimental animal models. Pharmacologia. 2011;2(6):172-7.

25. Monteiro EM, Chibli LA, Yamamoto CH, Pereira MC, Vilela FM, Rodarte MP, et al. Antinociceptive and anti-inflammatory activities of the sesame oil and sesamin. Nutrients. 2014;6(5):1931-44.

26. Gauthaman K. Nutraceutical value of sesame oil. Pharmacognosy Rev. 2009;3(6):264.

27. Sadeghi N, Oveisi MR, Hajimahmoodi M, Jannat B, Mazaheri M, Mansouri S. The contents of sesamol in Iranian sesame seeds. Iranian J Pharm Res. 2010:101-5.

28. Reiser MC. Comparing the visual analog scale and verbally administered numeric rating scale in traumatic versus non-traumatic causes of pain in an academic medical level 1 trauma emergency department.United States: The University of Toledo;2010.

29. Eftekhar Sadat B, Khadem Haghighian M, Alipoor B, Malek Mah davi A, Asghari Jafarabadi M, Moghaddam A. Effects of sesame seed supplementation on clinical signs and symptoms in patients with knee osteoarthritis. Int J Rheum Dis. 2013;16(5):578-82.

30. Nekuzad N, Ashke Torab T, Mojab F, Alavi-Majd H, Azadeh P, Ehtejab G. Effect of external use of sesame oil in the prevention of chemotherapy-induced phlebitis. Iran J Pharm Res. 2012;11(4):1065-71.

31. Newman DJ, Cragg GM. Natural products as sources of new drugs over the 30 years from 1981 to 2010. J Nat Prod. 2012;75(3):311-35.

32. Hirsch T, Ashkar W, Schumacher O, Steinstraesser L, Ingianni G Cedidi C. Moist exposed burn ointment (mebo) in partial thickness burns. Eur J Med Res. 2008;13:505-10.

33. Rangkadilok N, Pholphana N, Mahidol C, Wongyai W, Saengsooksree K, Nookabkaew S, et al. Variation of sesamin, sesamolin and tocopherols in sesame (Sesamum indicum L.) seeds and oil products in Thailand. Food Chemistry. 2010;122(3):724-30.

34. Deciga-Campos M, Montiel-Ruiz RM, Navarrete-Vazquez G, LopezMunoz FJ. Palmitic acid analogues exhibiting antinociceptive activity in mice. Proc West Pharmacol Soc. 2007;50:75-7.

35. Carvalho RHR, Galvão EL, Barros JC, Conceição MM, Sousa EMBD Extraction, fatty acid profile and antioxidant activity of sesame extract (Sesamum Indicum L.). Braz J Chem Eng. 2012;29(2):409-20.

36. Kupeli E, Erdemoglu N, Yesilada E, Sener B. Anti-inflammatory and antinociceptive activity of taxoids and lignans from the heartwood of Taxus baccata L. J Ethnopharmacol. 2003;89(2 3):265-70. 